\title{
Non-relativistic Perpendicular Shocks in Young Supernova Remnants
}

\author{
M. Pohl*, V. Wieland, I. Rafighi \\ Institute of Physics and Astronomy, University of Potsdam, Karl-Liebknecht-Strasse 24/25, \\ 14476 Potsdam, Germany \\ DESY, Platanenallee 6, 15738 Zeuthen, Germany \\ E-mail: marpohl@uni-potsdam.de
}

\section{J. Niemiec, A. Bohdan, O. Kobzar}

Instytut Fizyki Jądrowej PAN, ul. Radzikowskiego 152, 31-342 Kraków, Poland

E-mail: Jacek.Niemiec@ifj.edu.pl

\section{K.-I. Nishikawa}

Department of Physics, University of Alabama in Huntsville, Huntsville, AL 35899, USA

For parameters that are applicable to the conditions at young supernova remnants, we present results of $2 \mathrm{D} 3 \mathrm{~V}$ particle-in-cell simulations of a non-relativistic plasma shock with a large-scale perpendicular magnetic field. We developed a new clean setup that uses the collision of two plasma slabs with different density and velocity, leading to the development of two distinctive shocks and a contact discontinuity without artificial transients that may limit the veracity of the simulation. The Alfvenic Mach number of both shocks is $M_{\mathrm{A}} \simeq 30$, whereas the sonic Mach numbers differ with values $M_{\mathrm{S}} \simeq 250$ and $M_{\mathrm{s}} \simeq 750$. Both the forward and the reverse shocks are mediated by a Weibel-like filamentation instability that produces mainly magnetic turbulence. We observe significant shock rippling and strong fluctuations in the turbulent shock structure, and also features of the shock self-reformation. Proton reflection at the shocks leads to shocksurfing acceleration that generates a moderate non-thermal tail in the particle spectra measured downstream, suggesting that few ions undergo more than one reflection cycle. Electrons are preaccelerated in a layer of Buneman waves at the shock foot, but are very efficiently isotropized upon passage through the shock ramp, and hence their downstream spectrum is quasi-thermal with high temperature. We note that electrons and ions show the same transverse drift in the ramp region, which is commensurate with $\mathbf{E} x \mathbf{B}$ drift, but not the gradient drift that is usually invoked for shock drift acceleration. Thus, electrons loose energy by drifting. We discuss the impact of our findings on pre-acceleration of electrons at high-Mach-number perpendicular shocks and their injection into diffusive shock acceleration. First results of the studies of oblique quasiperpendicular shocks will also be presented.

The 34th International Cosmic Ray Conference,

30 July- 6 August, 2015

The Hague, The Netherlands

\footnotetext{
* Speaker.
} 


\section{Introduction}

Collisionless shocks in space are sites of efficient particle acceleration. Relevant for the process are the structure of the shocks, the electromagnetic fields at them, and the local preacceleration processes that separate particles from the quasithermal bulk. The shock structure is typically driven by ions and, therefore, has ion length scales. Electrons have small plasma and gyration scales compared with ions, and so electron pre-acceleration is a particularly interesting problem.

Studying electron acceleration requires that electron scales be resolved, and so we conduct particle-in-cell (PIC) simulations in 2D3V configuration, i.e. allowing gradients in 2 dimensions but following all 3 vector components. Hybrid simulations [1] permit studying the behavior on longer time scales and large spatial scales, but provide no information on electron dynamics. We here report on simulations of strictly perpendicular $\left(\theta_{B n}=90^{\circ}\right)$ shocks.

In contrast to earlier studies $[2,3,4,5,6,7,8]$, we concentrate on a parameter regime typical of young SNRs, as opposed to, e.g., heliospheric conditions, implying large sonic and Alfvénic Mach numbers, $M_{\mathrm{S}}$ and $M_{\mathrm{A}}$. We follow the shock evolution for 20 ion cyclotron times, $t \Omega_{i}=20$, considerably longer than other published PIC studies. We have also introduced a setup that minimizes artificial electromagnetic transients during shock launching, that may influence the upstream medium. This new method is physically more accurate as it avoids sharp gradients in the motional electric fields which act as an artificial dipole antenna and thereby emit a strong electromagnetic pulse whose presence in the system may limit the veracity of the simulation.

\section{Simulation Setup}

The numerical grid is initially filled with two plasma slabs of uniform density that are separated by a void and move towards each other. Once they collide, a system of two shocks and the CD is formed. We establish in the entire simulation box a homogeneous magnetic field $\mathbf{B}_{0}$ perpendicular to the streaming direction of the plasma beams. The magnetic field is meant to be frozen into the moving plasma, i.e. in the rest frame of the plasma $\mathbf{E}^{\prime}=0$ and in the simulation frame $\mathbf{E}=-\mathbf{v} \times \mathbf{B}^{\prime}$. This motional electric field has opposing signs in the two plasmas, which is illustrated in Figure 1. Without further modification, this setup would lead to a large value of $\nabla \times \mathbf{E}$ in the middle of the simulation box, that through the corresponding $\partial \mathbf{B} / \partial t$ would induce an electromagnetic transient that may limit the veracity of the simulation.

We developed a setup that avoids this artificial antenna effect by implementing a transition zone in the plasma-free area between the two slabs. A spatial gradient is imposed in the perpendicular magnetic-field components $B_{y}$ and $B_{z}$ ahead of the colliding plasma slabs with corresponding

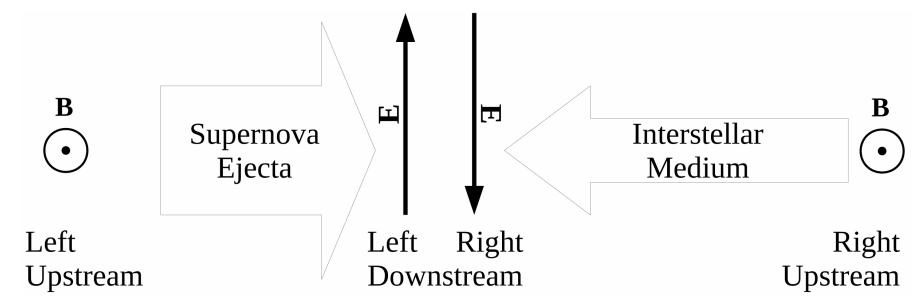

Figure 1: Illustration of the setup for a perpendicular shock showing the directions of motion of the two plasma slabs and the motional electric fields for a magnetic field oriented out of the plane of the figure. 


\begin{tabular}{lrr}
\hline & Left: reverse shock & Right: forward shock \\
\hline Skin length & $\lambda_{s e, \mathrm{~L}}=7.9 \Delta$ & $\lambda_{s e, \mathrm{R}}=25 \Delta$ \\
\hline Thermal speed, $v_{e, \mathrm{th}}$ & $0.002 \mathrm{c}$ & $0.002 \mathrm{c}$ \\
\hline Streaming speed & $v_{\mathrm{L}, x}=0.0354 c$ & $v_{\mathrm{R}, x}=-0.354 c$ \\
\hline Alfvén speed & $v_{\mathrm{A}, \mathrm{L}}=0.00447 c$ & $v_{\mathrm{A}, \mathrm{L}}=0.0142 c$ \\
\hline Shock speed & $v_{\mathrm{sh}, \mathrm{L}}=-0.127 c$ & $v_{\mathrm{sh}, \mathrm{R}}=0.39 c$ \\
\hline Mach number $M_{\mathrm{A}}$ & 28.5 & 27.6 \\
Mach number $M_{\mathrm{S}}$ & 252 & 755 \\
\hline
\end{tabular}

Table 1: Basic parameters of the double-shock simulation and derived shock properties. The left plasma slab has 10 times the density of the right plasma slab.

tapering of the motional electric field. This setup implies a nonzero $\nabla \times \mathbf{B}$ which is compensated by a current sheet in which ions drift relative to the electrons. We have verified that the new setup is stable over a sufficiently long time.

For ease of comparison with simulations of parallel shocks, we basically used the same simulation parameters as for simulation run M1 in [9]. All parameters are chosen such that our simulation results may be applied to plasma shocks formed at young supernova remnants. The two plasmas are composed of equal numbers of electrons and ions, but have different densities with a density ratio 10 , and hence their plasma frequencies differ by a factor $\sqrt{10} \simeq 3.1$. We use ten particles per cell per particle species for both plasma slabs and assign statistical weights to the tenuous-plasma particles to establish the intended density ratio. In order to resolve the characteristic length scales of both electrons and ions in our simulations, we choose a reduced mass ratio of $m_{i} / m_{e}=50$. The initially homogeneous magnetic field has equally large components in $y$ - and $z$-direction. The transverse size of the simulation box is $L_{y}=324.1 \lambda_{s e}=42.9 \lambda_{s i}$ with periodic boundary conditions. The main simulation parameters are summarized in Table 1 .

The simulation was performed with a modified version of the TRISTAN code [10], which was adapted to work in 2D3V and parallelized using MPI [11]. Other modifications include a fourthorder finite-difference time-domain field-pusher with Friedman filter [12] that eliminates numerical Čerenkov radiation, and an EoM integrator [13] that achieves better energy conservation and less numerical self-heating.

\section{Simulation results}

In the course of the simulation, the shocks move on average with the shock speed quoted in Table 1. Although the shock never completely disappears, a self-similar cyclic evolution is evident. Self-repeating reformation phases occur with a period of approximately $1.5 \Omega_{\mathrm{i}}^{-1}$ and are marked by positional shifts of the shock ramp with the enhancements in plasma density at the shock, and extensions of the filamentary region in the shock foot. This so-called shock reformation arises because specular ion reflection from the shock ramp is not a continuous process.

Figure 2 presents the structure of the forward shock at the end of the simulation at time $t=$ $20 \Omega_{\mathrm{i}}^{-1}$. Shock-reflected ions at supercritical perpendicular shocks trigger the growth of Buneman modes in the foot of the shock which subsequently heat the incoming electrons and can be seen

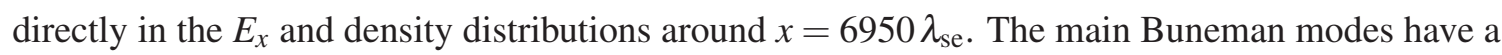


wavenumber as predicted by linear theory,

$$
k_{\|} \simeq \frac{c}{v_{\text {rel }}} \frac{1}{\lambda_{\text {se }, \text { local }}} \simeq 2.5 \frac{1}{3.1 \lambda_{\text {se }}} \simeq 0.8 \frac{1}{\lambda_{\text {se }}},
$$

The relative velocity of incoming electrons and reflected ions must be larger than the thermal speed of the electrons which leads to the condition [7]
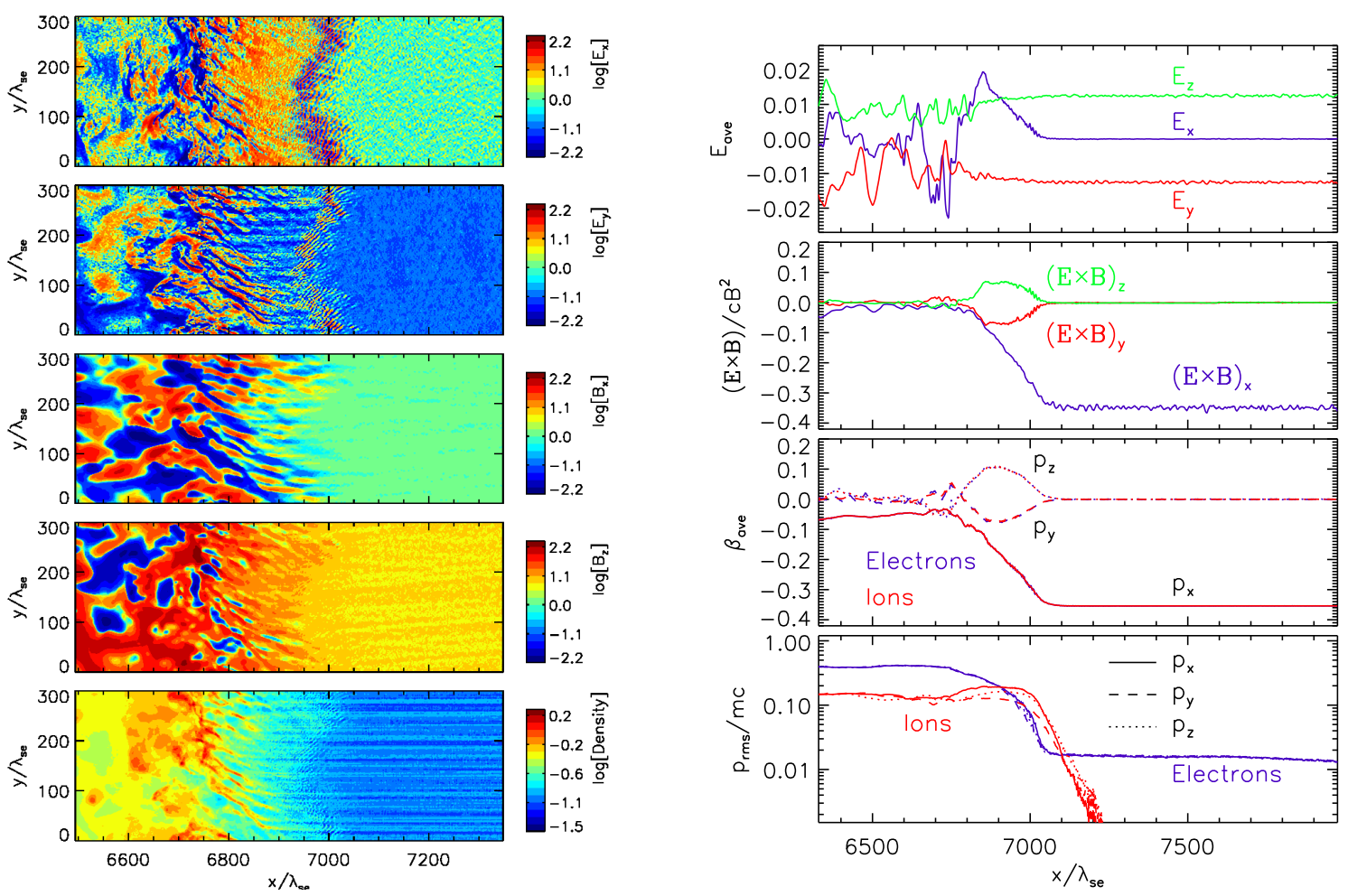

Figure 2: Left: Structure of the forward shock at the end of the simulation at time $t=20 \Omega_{\mathrm{i}}^{-1}$. Shown are from top to bottom the field components $E_{x}, E_{y}, B_{x}$, and $B_{z}$, followed by the electron density. As in earlier plots, we use a sign-preserving logarithmic scale for the field amplitudes. Right: Moments of the phasespace distribution of electrons and ions in comparison to the electric-field components and the components of $\mathbf{E} \times \mathbf{B}$ drift. All quantities are averaged over the $y$-coordinate. For ease of comparison with the drift speed we plot the average motion as velocity $\beta=p / m c\left(1+p^{2}\right)$.

$$
M_{\mathrm{s}} \geq \frac{1+\alpha}{2} \sqrt{\frac{m_{\mathrm{i}}}{m_{\mathrm{e}}}} \sqrt{\frac{T_{\mathrm{e}}}{T_{\mathrm{i}}}}
$$

where $\alpha$ denotes the density ratio of reflected and incoming ions. Originally this condition was written in terms of the Alfvénic Mach number and the electron plasma beta, which we consider not helpful because the Alfvén speed cancels in that expression. The total convertable electron drift energy density $[14,7]$, when turned into electron heat, gives

$$
\delta p_{\mathrm{x}, \mathrm{y}, \mathrm{z}} \simeq \frac{1}{\sqrt{3}} \frac{1}{1+\alpha} \frac{v_{\mathrm{sh}}}{c}\left(\frac{m_{\mathrm{e}}}{m_{\mathrm{i}}}\right)^{\frac{1}{6}} m_{\mathrm{e}} c \simeq 0.1 m_{\mathrm{e}} c,
$$

which is close to the observed spread of the $p_{x}$ phase-space component shown in the right panel of Fig. 2. The Alfvénic Mach number is too large, or the mass ratio between ions and electron 
too small, for Whistler waves to be generated in the foot region [15], that in low-Mach-number simulations were observed to energize electrons [6].

In the shock ramp and further downstream the electric field shows large fluctuations. Similar variations are seen in the magnetic field, but already after smoothing over scales smaller than the electron Larmor radius the $\mathbf{E} \times \mathbf{B}$ drift speed has a rather smooth distribution. In Fig. 2 the shock ramp is clearly delineated by the gradient in $(E \times B)_{x}$ between $x \simeq 6800 \lambda_{\text {se }}$ and $x \simeq 7000 \lambda_{\text {se }}$.

A gradient drift is also expected and should lead to shock-drift acceleration $[16,17]$, henceforth called SDA. Written in the downstream frame, the global gradient of the magnetic field near a nonrelativistic shock front causes a particle of mass $m$ and charge $q$ to drift with velocity [8]

$$
v_{\mathrm{gd}}=\frac{p_{\perp}^{2}}{2 m q B} \frac{\mathbf{B} \times \nabla B}{B^{2}}=\frac{\operatorname{sgn}(q) p_{\perp}^{2}}{2 m m_{i} \Omega_{i}} \frac{\mathbf{B} \times \nabla B}{B^{2}} \Rightarrow\left|v_{\mathrm{gd}}\right| \lesssim \frac{3}{16} \frac{m}{m_{i}} v_{\mathrm{sh}} \simeq 0.075 c \frac{m}{m_{i}}
$$

where $p_{\perp}$ is the momentum perpendicular to the magnetic field. In the last step, we have used for the gradient scale $v_{\mathrm{sh}} / \Omega_{i}$ and, on account of momentum conservation, $p_{\perp}^{2} \simeq(3 / 8) m^{2} v_{\mathrm{sh}}^{2}$.

Important is the dependence on the charge $\operatorname{sign}, \operatorname{sgn}(q)$, which mandates that electrons and ions drift in opposite directions. The observed mean perpendicular velocity, $\beta_{\text {ave }}=v_{\text {ave }} / c$, follows closely that expected from $\mathbf{E} \times \mathbf{B}$ drift. There is no discernible difference in the flow of electrons and ions, indicating the absence of a significant gradient drift. In fact, the large fluctuations in the electric and magnetic field play havoc with the gradient drift, because small-scale structures provide by far dominant contribution to $\nabla B$. We could not find an averaging scheme that permits extracting from the simulation data a gradient drift corresponding to that calculated in Eq. 3.4.

We also do not see any evidence for spontaneous turbulent reconnection in the ramp that was reported by others [18]. They noted a chain of magnetic islands coinciding with spikes in electron density resembling structures seen in dedicated simulations of turbulent reconnection. There, the neutral lines appear to break up into contracting magnetic islands, in which trapped electrons are accelerated through a Fermi-like process [19]. The filaments in our simulations are only weakly modulated on a scale similar to that of the Buneman waves in the foot, that the electrons passed through on their way up the ramp. Further modulation arises from filament mergers, but the amplitudes are moderate and they also do not align into a chain of magnetic islands.

In the ramp region a nearly perpendicular electromagnetic mode is evident in the field components shown in Figure 2. Similar filaments were observed in other simulations [20] and identified as current filaments resulting from the Weibel-type instabilities that were shown to mediate high-speed nonrelativistic unmagnetized and weakly magnetized parallel shocks [21,9]. After correcting for shock rippling, we conducted a Fourier analysis and indeed found strong evidence of filamentation in $\mathbf{j}_{x}$ and $\mathbf{j}_{z}$ (out of plane) that is carried exclusively by incoming particles.

Let us now turn to particle spectra. In a collisionless environment, isotropization and relaxation to near-Maxwellian distribution functions is achieved through interactions with plasma turbulence [22]. Figure 3 shows particle distributions in kinetic energy, $E_{\text {kin }}=(\gamma-1) m_{l} c^{2}\left(m_{1}=m_{\mathrm{e}}, m_{\mathrm{i}}\right)$ downstream of the forward and the reverse shock, respectively, at $t=20 \Omega_{\mathrm{i}}^{-1}$. The spectra are calculated in the downstream rest frame. We selected slices sufficiently far downstream, that the plasma is homogeneous and free of particles that penetrated the contact discontinuity. Both the ion and electron distributions are isotropic and display no large-scale bulk motion. 

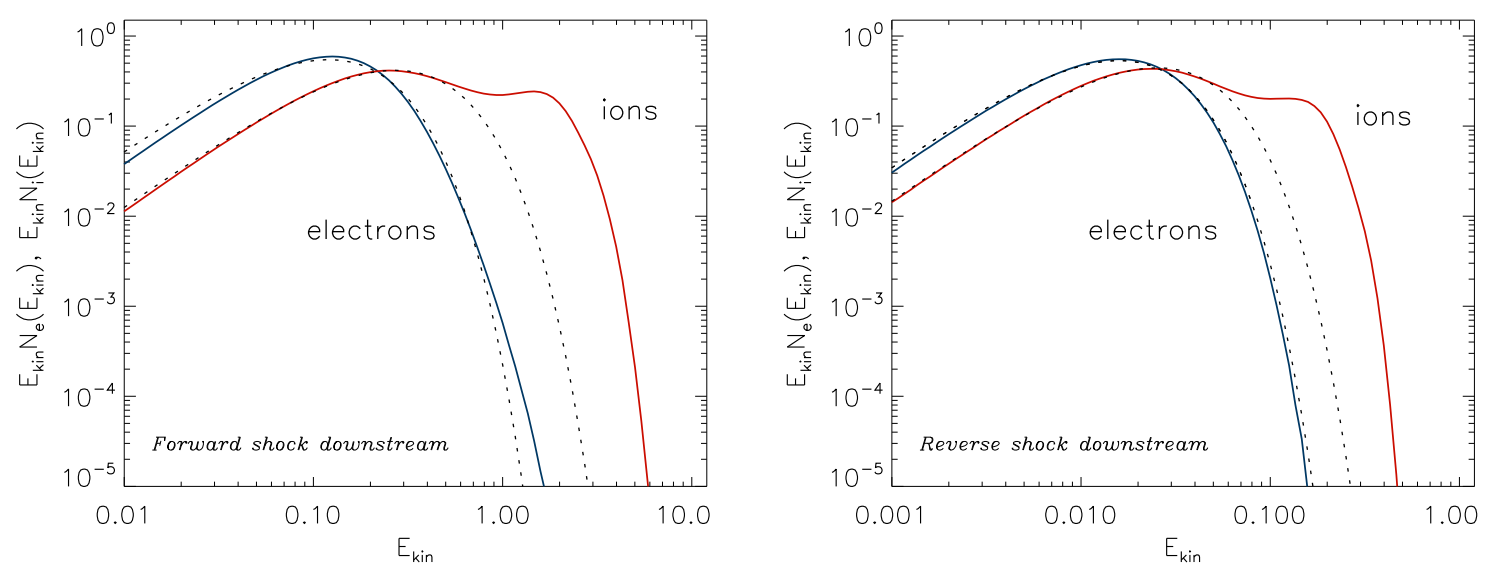

Figure 3: Left: Kinetic-energy spectra of electrons (blue line) and ions (red line) at time $t=20 \Omega_{\mathrm{i}}^{-1}$ and the region $x \approx 5000-5700 \lambda_{\text {se }}$ far downstream of the forward shock at $x \approx 6800 \lambda_{\text {se. }}$ The spectra are calculated in the downstream rest frame. They are normalized and expressed in simulation units, in which $m_{e} c^{2}=0.25$. The dotted lines indicate fits of a relativistic Maxwellian. Right: Corresponding particle spectra in the region $x \approx 2910-2980 \lambda_{\text {se }}$ far downstream of the reverse shock at $x \approx 2800 \lambda_{\mathrm{se}}$.

For both the forward and the reverse shock, the distributions of electrons appear to be quasithermalized and well reproduced by relativistic Maxwellians. A Maxwellian fit to the bulk of the ion distributions suggests a temperature ratio $T_{\mathrm{i}} / T_{\mathrm{e}} \approx 2$. In addition, the ion spectra exhibit a significant supra-thermal tail. With the exception of electrons downstream of the reverse shock, whose temperature slightly increases with distance from the shock, particle spectra do not show significant evolution. Their distributions at fixed distance from the shock are also approximately steady in the time for $t \approx 5-20 \Omega_{\mathrm{i}}^{-1}$, which demonstrates that the system has attained a quasiequilibrium within the runtime of our simulations.

It is known that the formation of supra-thermal tails in the ion distribution at perpendicular shocks can result from shock-surfing acceleration, in which shock-reflected particles performing half a gyro cycle upstream increase their energies through motion along the convective electric field. The maximum ion energy is roughly constant in our simulation, which suggests that a typical particle needs only a few gyro cycles to traverse the shock potential and be transmitted downstream.

There are two obvious differences between published simulations showing efficient electron acceleration and studies yielding little electron energization. First, it appears that strong acceleration is seen when the large-scale magnetic field is assumed to be strictly out of plane, as in [7, 23]. Then, these authors tend to use a larger ion-to-electron mass ratio of 100-225 compared to 25-50 as in [20] and this paper. One consequence is a stronger magnetization of electrons in the former simulations. It is unclear to what degree this correlation signifies causation. Reconnection is seen only in a simulation with in-plane magnetic field [18], but not with our setup with $45^{\circ}$ configuration.

\section{Summary and discussion}

We have performed 2D3V PIC simulations of non-relativistic plasma collisions with perpendicular large-scale magnetic field. Our results can be summarized as follows: 
1) Our newly developed setup leads to the creation of a very clean perpendicular shock without artificial transients that may limit the veracity of the simulation. Eventually, a double-shock structure evolves within a few ion cyclotron times, $\Omega_{\mathrm{i}}^{-1}$. The shock transition is mediated by Weibel-type filamentation instabilities that lead to the development of current filaments and magnetic turbulence.

2) The surface of both shocks is clearly rippled. In terms of the local plasma scale, $\lambda_{\text {si,loc }}$, the amplitude of rippling is significantly smaller, and the wavelength slightly smaller, at the forward shock that has a sonic Mach number a factor of three higher than that of the reverse shock. This affects the visibility of shock reformation, which is washed out in $y$-averaged density profiles of the reverse shock. Generally, the periodicity of ion reflection that drives shock reformation is locally modified by the non-coherent evolution of current filaments across the shock. The period of reformation is similar at both shocks with $\sim 1.5 \Omega_{\mathrm{i}}^{-1}$.

3) We do not find any evidence for gradient drift at the shock, which would be an integral part of SDA. It is not possible to reconstruct from the available electromagnetic field data a uniform drift direction, because the local gradients in the magnetic field are much larger than the global gradient across the shock. We also do not find any indication of counterstreaming electrons and ions along the shock surface. In fact, the bulk motion of electrons and ions is commensurate with $\mathbf{E} \times \mathbf{B}$ drift in direction and amplitude.

4) Downstream of both shocks the electrons are well described by relativistic Maxwellians, suggesting that turbulence has been very efficient in relaxing the electron distribution function. Ion spectra are composed of a quasi-thermal bulk with $T_{\mathrm{i}} / T_{\mathrm{e}} \approx 2$ and a supra-thermal tail. We do not observe spectral variations with either distance from the shock or simulation time for $t \approx$ $5-20 \Omega_{\mathrm{i}}^{-1}$, suggesting a quasi-equilibrium in the system. The supra-thermal ions appear to result from shock-surfing acceleration. The constancy of the maximum ion energy suggests that their spending more than a few gyro cycles in the shock region is exceptional. Electron heating arises from Buneman modes in the shock foot. Their amplitude is not high enough to prevent escape of relativistic electrons, and so we observe heating of the bulk as opposed to the creation of a spectral tail. There is no evidence of turbulent reconnection that was recently claimed to cause efficient electron energization [18]. The inefficient electron acceleration observed by us for low plasma beta and by [20] for high plasma beta suggests that the plasma beta is not the deciding factor for the generation of a signicant non-thermal electron population. The configuration of the large-scale magnetic field in 2D3V simulations may have an impact, as recent simulations showing significant tails in electron spectra have an orientation strictly out of plane [7, 23], although our setup with $45^{\circ}$ orientation to the simulation plane should help suppressing such effects. Another possibility is that the ion-to-electron mass ratio in the simulation plays a role, as electron acceleration is often observed in simulations with $m_{i} / m_{e}$ in the range 100-225 compared to 25-50 as in [20] and this paper. There may be additional factors in the microphysics of high-Mach-number shocks mediated by filamentation that limit the amplitude of Buneman waves or prevent return of electrons to the foot region, resulting in a small number of supra-thermal electrons.

Acknowledgements: This work used the Extreme Science and Engineering Discovery Environment (XSEDE), which is supported by National Science Foundation grant number ACI1053575, computing resources provided by the Texas Advanced Computing Center (TACC) at The University of Texas at Austin , and HPC resources provided by The North-German Supercom- 
puting Alliance (HLRN). V.W., M.P. and I.R. acknowledge support through grants PO 1508/11 and PO 1508/1-2 of the Deutsche Forschungsgemeinschaft. The work of J.N. is supported by Narodowe Centrum Nauki through research projects DEC-2011/01/B/ST9/03183 and DEC2013/10/E/ST9/00662. K.N. is supported by NSF AST-0908040, NNX12AH06G, NNX13AP21G, and NNX13AP14G.

\section{References}

[1] Caprioli, D., \& Spitkovsky, A. 2014, ApJ, 783, 91

[2] Umeda, T., Yamao, M., \& Yamazaki, R. 2008, ApJ Lett., 681, L85

[3] Amano, T., \& Hoshino, M. 2007, ApJ, 661, 190

[4] Amano, T., \& Hoshino, M. 2009, ApJ, 690, 244

[5] Amano, T., \& Hoshino, M. 2010, Phys. Rev. Lett., 104, 181102 (1

[6] Riquelme, M. A., \& Spitkovsky, A. 2011, ApJ, 733, 63 (1

[7] Matsumoto, Y., Amano, T., \& Hoshino, M. 2012, ApJ, 755, 109

[8] Guo, X., Sironi, L., \& Narayan, R. 2014, ApJ, 794, 153

[9] Niemiec, J., Pohl, M., Bret, A., \& Wieland, V. 2012, ApJ, 759, 73

[10] Buneman, O. 1993, Computer Space Plasma Physics, ed. H. Matsumoto \& Y. Omura (Terra Scientific Publishing Company), 67-84

[11] Niemiec, J., Pohl, M., Stroman, T., \& Nishikawa, K.-I. 2008, ApJ, 684, 1174

[12] Greenwood, A. D., Cartwright, K. L., Luginsland, J. W., \& Baca, E. A. 2004, J. Comp. Phys., 201, 665

[13] Vay, J.-L. 2008, Phys. Pl., 15, 056701 (1

[14] Ishihara, O., Hirose, A., \& Langdon, A. B. 1980, Phys. Rev. Lett., 44, 1404

[15] Matsukiyo, S., \& Scholer, M. 2006, J. Geo. Res., 111, 6104

[16] Krauss-Varban, D., Burgess, D., \& Wu, C. S. 1989, J. Geo. Res., 94, 15089

[17] Krauss-Varban, D., \& Wu, C. 1989, J. Geo. Res., 94, 15367

[18] Matsumoto, Y., Amano, T., Kato, T. N., \& Hoshino, M. 2015, Science, 347, 974

[19] Drake, J. F., Swisdak, M., Che, H., \& Shay, M. A. 2006, Nature, 443, 553

[20] Kato, T. N., \& Takabe, H. 2010, ApJ, 721, 828

[21] Kato, T. N., \& Takabe, H. 2008, ApJ Lett., 681, L93

[22] Bret, A. 2015, arXiv:1502.00626

[23] Matsumoto, Y., Amano, T., \& Hoshino, M. 2013, Phys. Rev. Lett., 111, 215003 